Jeroen J. G. Geurts, MSc

Petra J. W. Pouwels, PhD

Bernard M. J. Uitdehaag, MD, PhD

Chris H. Polman, MD, PhD

Frederik Barkhof, MD, PhD

Jonas A. Castelijns, MD,

PhD

Published online

10.1148/radiol.2361040450

Radiology 2005; 236:254-260

Abbreviations:

$\mathrm{Cl}=$ confidence interval

CSF $=$ cerebrospinal fluid

$\mathrm{DIR}=$ double inversion recovery

FLAIR = fluid-attenuated inversion

recovery

$\mathrm{SE}=$ spin echo

$3 \mathrm{D}=$ three-dimensional

${ }^{1}$ From the Departments of Radiology (J.J.G.G., F.B., J.A.C.), Neurology (B.M.J.U., C.H.P.), and Physics and Medical Technology (P.J.W.P.), MR Center for MS Research, VU University Medical Center, De Boelelaan 1117, 1081 HV Amsterdam, the Netherlands. Received March 10, 2004; revision requested May 21; final revision received September 2; accepted September 29. J.J.G.G. supported by grant 00-427 from the Stichting MS Research, Voorschoten, the Netherlands. Address correspondence to J.J.G.G. (e-mail: j.geurts@vumc.nI).

Authors stated no financial relationship to disclose.

\section{Intracortical Lesions in Multiple Sclerosis: Improved Detection with 3D Double Inversion-Recovery MR Imaging 1}

PURPOSE: To prospectively compare the depiction of intracortical lesions by using multislab three-dimensional (3D) double inversion-recovery (DIR), multislab 3D fluid-attenuated inversion-recovery (FLAIR), and T2-weighted spin-echo (SE) magnetic resonance (MR) imaging in patients with multiple sclerosis.

MATERIALS AND METHODS: Local ethics review board approval and informed consent were obtained. Conventional T2-weighted SE and multislab 3D FLAIR and DIR images were acquired in 10 patients with multiple sclerosis (five women, five men) and 11 age-matched healthy control subjects (seven women, four men). Mean age was 40 years (range, 25-54 years) in patients and 34 years (range, 24-55 years) in control subjects. Lesions were classified according to seven anatomic regions: intracortical, mixed white matter-gray matter, juxtacortical, deep gray matter, periventricular white matter, deep white matter, and infratentorial lesions. The numbers of lesions per category were compared between techniques (Dunnettcorrected analysis of variance). Gain or loss (with $95 \%$ confidence intervals [Cls]) of numbers of lesions detected at 3D DIR imaging was calculated in comparison with those detected at T2-weighted SE and 3D FLAIR imaging.

RESULTS: Total number of lesions did not differ between 3D DIR and 3D FLAIR sequences, but the 3D DIR sequence showed a gain of $21 \%(95 \% \mathrm{CI}: 4 \%, 41 \%)$ in comparison with the T2-weighted SE sequence. Because of high gray matter-white matter contrast, DIR images depicted more intracortical lesions (80 lesions in 10 patients) than both SE (10 lesions) and FLAIR (31 lesions) images; gains with DIR were $538 \%$ (95\% Cl: $191 \%, 1297 \%)$ and $152 \%$ (95\% Cl: $15 \%, 453 \%)$ compared with SE and FLAIR, respectively. Only four intracortical lesions were detected in control subjects. Also, DIR imaging enabled a better definition of mixed white matter-gray matter lesions because of greater contrast between the lesion and its surroundings.

CONCLUSION: MR imaging with 3D DIR enables increased intracortical lesion detection in the multiple sclerosis brain, as well as improved distinction between juxtacortical and white matter-gray matter lesions.

○ RSNA, 2005

\section{Author contributions:}

Guarantors of integrity of entire study, J.J.G.G., P.J.W.P., J.A.C.; study concepts and design, all authors; literature research, J.J.G.G.; clinical studies, J.J.G.G., J.A.C.; data acquisition and analysis/interpretation, all authors; statistical analysis, J.J.G.G., P.J.W.P., B.M.J.U., J.A.C.; manuscript editing, J.J.G.G., P.J.W.P., B.M.J.U., F.B., J.A.C.; manuscript preparation, definition of intellectual content, revision/review, and final version approval, all authors o RSNA, 2005
Multiple sclerosis is an inflammatory demyelinating disease of the central nervous system that usually affects young adults and leads to chronic invalidism. While the disease typically affects the (periventricular) white matter, the role of the gray matter in the pathophysiology of multiple sclerosis has gained increasing attention over the last few years (1-9). Results of histopathologic studies have shown that a substantial portion of the total cerebral lesion load in multiple sclerosis is located within the cerebral cortex or at the border between the cortex and subcortical white matter $(4,10-12)$. It has also been shown that extensive subpial demyelination exists in the multiple sclerosis brain, with lesions extending from the pia mater downward over large areas of cortical gray matter (13). 
Since abnormalities in cortical gray matter have been correlated with both physical and neuropsychologic deficits in patients with multiple sclerosis (14-16), it is essential to create a better understanding and a more accurate estimation of the amount of gray matter lesions in vivo.

Unfortunately, presently available magnetic resonance (MR) imaging techniques are usually not optimal for detecting cortical lesions (4). Authors of previous studies have reported increased cortical and/ or subcortical lesion detection by using two-dimensional and three-dimensional (3D) fluid-attenuated inversion-recovery (FLAIR) MR imaging (14,17-20). However, the exact anatomic border between the cortex and subcortical white matter may be hard to determine on a FLAIR MR image, which creates difficulties in judging whether lesions are juxtacortical, mixed white matter-gray matter, or intracortical. With a double inversionrecovery (DIR) MR sequence, inversion times can be selected such that suppression of the signals from both white matter and cerebrospinal fluid (CSF) is achieved, yielding images that show superior delineation of gray matter; thus, the contrast is due to differences in T1 relaxation times between gray matter and CSF, as well as between gray and white matter. This method has been described before as a helpful tool in the detection of lesions in multiple sclerosis (21). In a comparison between twodimensional DIR MR imaging and twodimensional FLAIR MR imaging, it was concluded that two-dimensional DIR imaging might be preferential when infratentorial lesions (either neoplastic or demyelinating) and abnormalities with only slightly prolonged T2 values (eg, cortical lesions) were concerned (22).

Recently, a multislab 3D DIR sequence was developed (23) that combined the aforementioned selective contrast with high spatial resolution both in plane and through plane. Thus, the purpose of our study was to prospectively compare the depiction of intracortical lesions by using a multislab 3D DIR, a multislab 3D FLAIR, and a T2-weighted spin-echo (SE) MR imaging sequence in patients with multiple sclerosis.

\section{MATERIALS AND METHODS}

\section{Patients and Control Subjects}

Ten patients with chronic, clinically definite (24) multiple sclerosis (secondary progressive in eight patients, relaps-

\section{TABLE 1}

Sequence Parameters for Multislab 3D DIR and FLAIR and Conventional T2-weighted SE MR Imaging

\begin{tabular}{|c|c|c|c|}
\hline Parameter & 3D DIR & 3D FLAIR & T2-weighted SE \\
\hline Repetition time (msec) & 7100 & 6500 & 2690 \\
\hline Effective echo time (msec) & 99 & 119 & 45,90 \\
\hline Inversion time (msec) & $330,2480^{*}$ & 2200 & $\cdots$ \\
\hline Bandwidth (Hz per pixel) & 257 & 186 & 78 \\
\hline Turbo factor & 37 & 37 & $\ldots$ \\
\hline No. of sections ${ }^{\dagger}$ & $\ldots$ & $\ldots$ & $2 \times 25$ \\
\hline No. of slabs ${ }^{\dagger}$ & $2 \times 4$ & $2 \times 6$ & $\ldots$ \\
\hline No. of sections per slab & 10 & 10 & $\cdots$ \\
\hline Section thickness (mm) & 1.8 & 1.25 & 3.0 \\
\hline Matrix size & $147 \times 256$ & $147 \times 256$ & $158 \times 256$ \\
\hline Field of view (mm) & $194 \times 310$ & $194 \times 310$ & $179 \times 260$ \\
\hline In-plane pixel size (mm) & $1.3 \times 1.2$ & $1.3 \times 1.2$ & $1.1 \times 1.0$ \\
\hline Acquisition time (min) & 15.3 & 14.1 & 14.2 \\
\hline
\end{tabular}

* The long inversion time is the duration between the two inversion pulses, and the short inversion time is the duration between the second inversion pulse and the excitation pulse.

$\dagger$ " $2 \times$ " indicates that the sequence was performed twice, in a section- or slab-interleaved manner.

$\ddagger$ With an additional $60 \%$ oversampling in the section-select direction.

ing-remitting in two patients; median Expanded Disability Status Scale score, 4.0; mean disease duration, 12 years) and 11 age-matched healthy control subjects were selected for this study (J.J.G.G., C.H.P.). Patients were randomly selected from a clinical database of multiple sclerosis. Health status of the control subjects was determined by evaluating their medical history. The study protocol was approved by the local ethics review board of the VU University Medical Center, and all subjects gave informed consent prior to examination. Mean age among the 10 patients (five men, five women) was 40 years (range, 25-54 years), and mean age among the 11 control subjects (four men, seven women) was 34 years (range, 24-55 years). No statistically significant differences in age or sex were found between patients and control subjects, as determined by means of the Student $t$ test.

\section{MR Image Acquisition}

Examinations were performed (J.J.G.G., P.J.W.P.) with a 1.5-T whole-body MR imager (Sonata; Siemens, Erlangen, Germany) by using a standard circularly polarized head coil. Standard dual-echo T2-weighted SE, 3D FLAIR, and 3D DIR MR images were acquired in random order. Sequence parameters are available in Table 1.

The multislab 3D FLAIR and 3D DIR methods are based on 3D turbo SE sequences preceded by one or two adiabatic inversion pulses, respectively, that selectively invert the imaging slabs $(20,23)$. To reduce acquisition times in comparison with those in previous studies, the turbo factor was increased for both 3D DIR and 3D FLAIR sequences at the expense of a loss in signal-to-noise and contrast-tonoise ratios. The 3D DIR and 3D FLAIR sequences contain a fat-saturation pulse, and an inferior saturation slab was applied to reduce flow artifacts for all sequences.

On the basis of the duration of the interleaved T2-weighted SE sequence, which is a standard method to measure numbers of lesions in multiple sclerosis research, the spatial resolution of the two 3D methods was adjusted to yield similar acquisition times that are acceptable for clinical research purposes. Thus, the voxel dimensions were $3.30 \mathrm{~mm}^{3}$ for T2weighted SE imaging, $1.95 \mathrm{~mm}^{3}$ for 3D FLAIR imaging, and $2.81 \mathrm{~mm}^{3}$ for 3D DIR imaging (Table 1).

\section{Image Analysis}

Two experienced neuroradiologists (J.A.C. and F.B., both with 12 years of neuroradiology experience) interpreted the images by consensus. Lesions in patients with multiple sclerosis and in control subjects were classified (J.J.G.G.) according to their location as intracortical, mixed white matter-gray matter, juxtacortical white matter (not entering the cortical gray matter), deep gray matter, periventricular white matter, deep white matter, or infratentorial. Possible artifacts were evaluated on 3D DIR MR images of healthy control subjects.

In patients, the contrast ratio was determined between gray or white matter and lesions. The contrast ratio was de- 

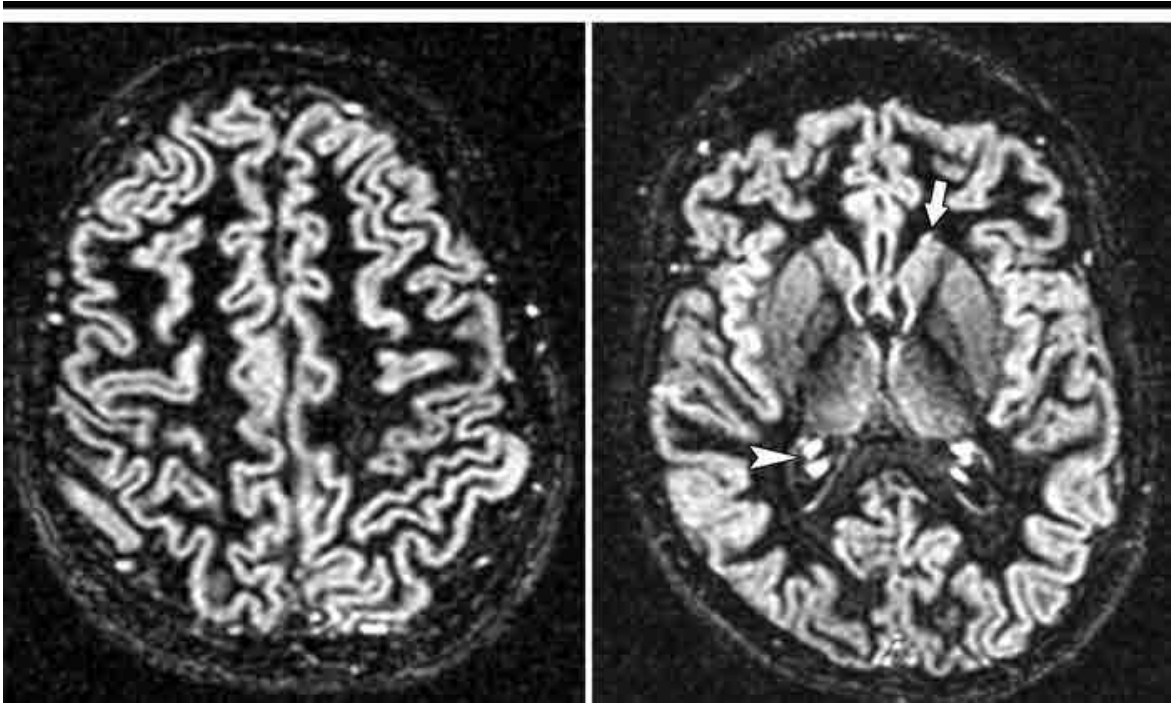

rected $P<.05$ was considered to denote a significant difference.

Correlations between disease duration of multiple sclerosis or Expanded Disability Status Scale and lesion scores (either total or specifically intracortical) were investigated by using Spearman correlation coefficient. All statistical analyses (J.J.G.G., P.J.W.P., B.M.J.U.) were performed by using SPSS version 11.0 (SPSS, Chicago, Ill) and Excel 2002 (Microsoft, Redmond, Wash).

\section{RESULTS}

\section{Control Subjects}

An example of 3D DIR MR imaging in a control subject is shown in Figure 1 . The 3D DIR images of the control subjects in this study revealed areas of high signal intensity that were comparable with the artifacts defined in 3D FLAIR studies (20). If present, these areas were located in the posterior fossa, the choroid plexus, and the periventricular white matter and periaqueductal brainstem tissue, most probably as a result of transependymal CSF effusion. However, these areas of high signal intensity were not considered diagnostically relevant, and CSF suppression was sufficient on both 3D FLAIR and 3D DIR MR images.

In control subjects $(n=11)$, T2weighted SE, 3D FLAIR, and 3D DIR images showed a total of 18,36 , and 45 lesions, respectively. It is noteworthy that $72(73 \%)$ of these 99 lesions were detected in one subject, a 55-year-old woman. In total, 77 (78\%) of 99 lesions in control subjects were located in the periventricular and deep white matter and were described to be most probably of vascular origin. No intracortical lesions were found with T2-weighted SE, one lesion was found with 3D FLAIR, and three lesions were found with 3D DIR MR imaging.

\section{Patients with Multiple Sclerosis}

In patients with multiple sclerosis, 1455 lesions were detected with T2weighted SE, 1714 lesions were detected with 3D FLAIR, and 1735 lesions were detected with 3D DIR MR imaging (Table 2). When considering lesion categories separately, 3D DIR imaging was superior in depicting intracortical lesions in comparison with both T2-weighted SE and 3D FLAIR MR imaging (Fig 2). On average, 3D DIR imaging depicted 7.0 more intracortical lesions per patient than did T2-weighted SE imaging. In comparison with 3D FLAIR imaging, 3D DIR imaging 
TABLE 2

Mean Numbers of Lesions Detected and Relative Comparison of 3D DIR versus T2-weighted SE and 3D FLAIR Imaging for Depicting Lesions

\begin{tabular}{|c|c|c|c|c|c|}
\hline \multirow[b]{2}{*}{ Region } & \multirow[b]{2}{*}{ T2-weighted SE* } & \multirow[b]{2}{*}{ 3D FLAIR* } & \multirow[b]{2}{*}{ 3D DIR* } & \multicolumn{2}{|c|}{ Relative Comparison (\%) ${ }^{\dagger}$} \\
\hline & & & & DIR vs T2 SE & DIR vs FLAIR \\
\hline Intracortical & $1.0 \pm 1.8$ & $3.1 \pm 4.0$ & $8.0 \pm 6.0$ & $538(191,1297)$ & $152(15,453)$ \\
\hline Mixed WM-GM & $5.9 \pm 6.3$ & $12 \pm 18$ & $16 \pm 18$ & $165(43,390)$ & $39(-25,157)$ \\
\hline Juxtacortical & $32 \pm 43$ & $12 \pm 10$ & $16 \pm 13$ & $-38(-64,6)$ & $23(-28,109)$ \\
\hline Deep GM & $4.0 \pm 5.0$ & $6.5 \pm 7.2$ & $6.8 \pm 6.3$ & $87(2,242)$ & $37(-25,151)$ \\
\hline Periventricular WM & $60 \pm 38$ & $69 \pm 39$ & $73 \pm 48$ & $16(-8,46)$ & $-2(-22,23)$ \\
\hline Deep WM & $33 \pm 15$ & $60 \pm 44$ & $42 \pm 19$ & $24(1,52)$ & $-25(-39,-8)$ \\
\hline Infratentorial & $10 \pm 11$ & $8.4 \pm 8.2$ & $12 \pm 10$ & $40(-25,164)$ & $72(-8,223)$ \\
\hline Overall & $146 \pm 108$ & $171 \pm 113$ & $174 \pm 110$ & $21(4,41)$ & $1(-14,17)$ \\
\hline
\end{tabular}

depicted an average of 4.9 more intracortical lesions per patient. The relative gain for 3D DIR imaging was 538\% (95\% CI: $191 \%, 1297 \%)$ with respect to T2weighted SE imaging and 152\% (95\% CI: $15 \%, 453 \%)$ with respect to 3D FLAIR imaging.

The largest number of mixed white matter-gray matter lesions was observed with 3D DIR imaging, followed by 3D FLAIR imaging and T2-weighted SE imaging. The relative comparison between 3D DIR and T2-weighted SE imaging revealed a mean gain of $165 \%$ (95\% CI: $43 \%, 390 \%)$ at 3D DIR imaging versus T2-weighted SE imaging. The highest numbers of juxtacortical lesions were scored on T2-weighted SE images, followed by 3D DIR images and 3D FLAIR images. As a consequence, the mean relative loss in lesions detected at 3D DIR imaging in comparison with T2-weighted SE imaging was estimated to be $38 \%$ (95\% CI: $-64 \%, 6 \%$ ). MR imaging with the 3D DIR sequence showed a mean gain over imaging with the 3D FLAIR sequence of $39 \%$ (95\% CI: $-25 \%, 157 \%$ ) for mixed white matter-gray matter and of $23 \%(-28 \%, 109 \%)$ for juxtacortical lesions. These lesion types are illustrated in Figure 3.

The 3D DIR and 3D FLAIR images depicted comparable numbers of deep gray matter lesions, with a relative gain of 87\% (95\% CI: 2\%, 242\%) when comparing 3D DIR images with T2-weighted SE images. For the remaining lesion categories, the mean relative comparisons between 3D DIR imaging and T2-weighted SE or 3D FLAIR MR imaging did not exceed 50\%, except for infratentorial lesions, for which there was a gain of $72 \%$ (95\% CI: $-8 \%, 223 \%)$ for 3D DIR imaging in comparison with 3D FLAIR imag-

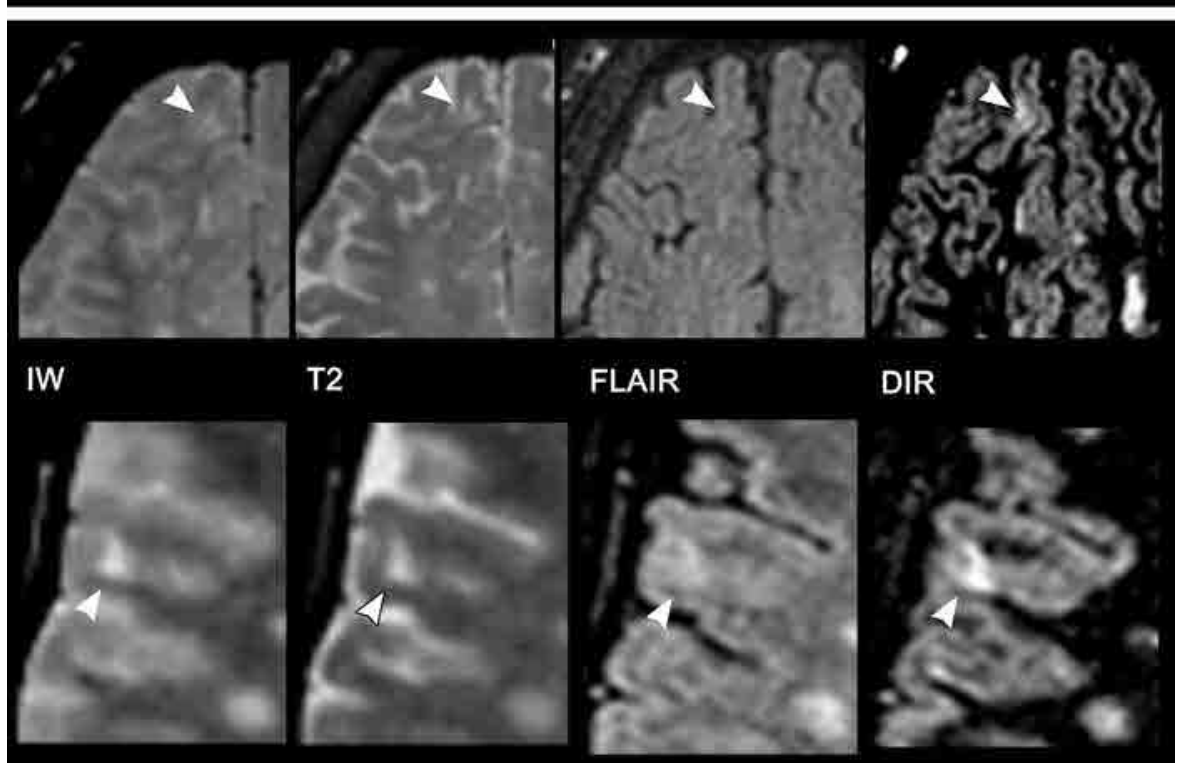

Figure 2. Transverse intermediate-weighted (IW), T2-weighted (T2), 3D FLAIR, and 3D DIR images of intracortical lesions. Top row: lesion (arrowhead) in the cortical gray matter, with a possible juxtacortical component; the intracortical lesion is particularly poorly visible on intermediate- and T2-weighted images, as well as on the FLAIR image, whereas it is depicted clearly on the DIR image. Bottom row (different patient): DIR image shows very good delineation of the intracortical lesion (arrowhead), which may be mistaken for a juxtacortical lesion or a partial volume artifact on the T2-weighted image and may even be missed on the FLAIR image. No contrast agent was used.

ing. A larger number of white matter lesions (periventricular and deep white matter) was detected by using 3D DIR in comparison with T2-weighted SE imaging. The mean relative gains were quite low (16\% and $24 \%$ for periventricular and deep white matter, respectively), reflecting the larger number of white matter lesions that are already detected on T2-weighted SE images.

Comparisons resulting in a relative loss of lesion detection at 3D DIR imaging were observed for 3D FLAIR imaging in the categories of deep white matter le- sions (-25\%; 95\% CI: $-39 \%,-8 \%)$ and periventricular lesions (-2\%; 95\% CI: $-22 \%, 23 \%$ ).

In patients with multiple sclerosis, no correlations were found between disease duration, the Expanded Disability Status Scale score, and numbers of lesions.

\section{Image Contrast}

The regions of interest that were selected for contrast measurements had mean areas of $128 \mathrm{~mm}^{2}$ (range, 35-318 $\mathrm{mm}^{2}$ ) for gray matter, $138 \mathrm{~mm}^{2}$ (range, 


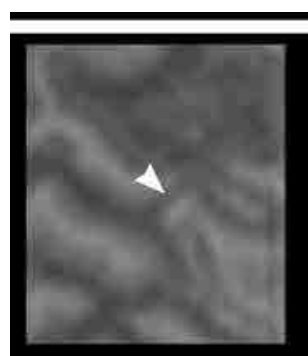

IW

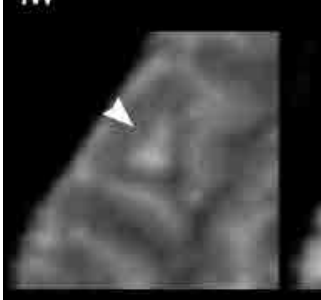

T2

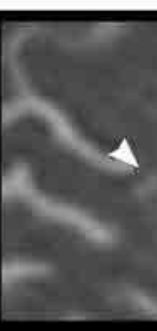

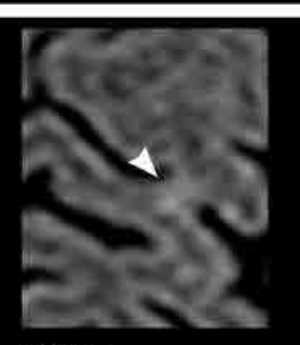

FLAIR

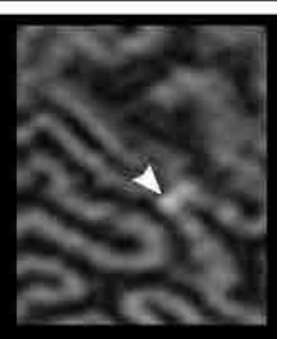

DIR

Figure 3. Transverse intermediate-weighted $(I W)$, T2-weighted (T2), 3D FLAIR, and 3D DIR images of mixed white matter-gray matter lesions (top and bottom rows represent two different patients). Whereas it may be hard to determine whether the lesion (arrowhead) in each patient is juxtacortical or mixed white matter and gray matter on T2-weighted and FLAIR images, DIR images show in more detail that these lesions also involve the cortical gray matter. No contrast agent was used.

\begin{tabular}{|c|c|c|c|}
\hline Parameter & 3D DIR & 3D FLAIR & T2-weighted SE \\
\hline \multicolumn{4}{|l|}{ Contrast ratio } \\
\hline Lesion-white matter & $11.9 \pm 2.8^{\star \dagger}$ & $0.98 \pm 0.14^{\dagger}$ & $0.52 \pm 0.10$ \\
\hline Lesion-gray matter & $0.60 \pm 0.27^{\dagger \ddagger}$ & $0.32 \pm 0.12$ & $0.19 \pm 0.07$ \\
\hline \multicolumn{4}{|l|}{ Contrast-to-noise ratio } \\
\hline White matter-gray matter & $20.7 \pm 5.1^{\ddagger}$ & $14.4 \pm 4.8^{\S}$ & $22.1 \pm 6.0$ \\
\hline White matter-CSF & $0.7 \pm 0.5^{\star \dagger}$ & $22.3 \pm 3.1^{\dagger}$ & $30.3 \pm 6.2$ \\
\hline Gray matter-CSF & $21.1 \pm 4.9^{\star \dagger}$ & $36.8 \pm 6.4^{\dagger}$ & $8.6 \pm 4.4$ \\
\hline Lesion-white matter & $33.0 \pm 7.6$ & $27.3 \pm 5.5$ & $40.2 \pm 9.4$ \\
\hline Lesion-gray matter & $13.0 \pm 5.5$ & $13.2 \pm 4.5$ & $19.0 \pm 7.3$ \\
\hline Lesion-CSF & $33.4 \pm 7.6^{* \dagger}$ & $49.8 \pm 7.6^{\dagger}$ & $11.7 \pm 8.4$ \\
\hline
\end{tabular}

Note.-Data are means \pm standard deviations. Two-tailed paired Student $t$ tests (Bonferroni corrected) were performed.

${ }^{*} P<.01$ in comparison with 3D FLAIR imaging.

$\dagger P<.01$ in comparison with T2-weighted SE imaging

$¥ P<.05$ in comparison with 3D FLAIR imaging.

$\S P<.05$ in comparison with T2-weighted SE imaging.

$18-470 \mathrm{~mm}^{2}$ ) for white matter, $41 \mathrm{~mm}^{2}$ (range, 11-206 $\mathrm{mm}^{2}$ ) for white matter lesions, and $1392 \mathrm{~mm}^{2}$ (range, 356-5081 $\mathrm{mm}^{2}$ ) for noise. Gray matter lesions have a signal intensity similar to that of white matter lesions but are much smaller. Therefore, white matter lesions were used to determine the signal intensity of lesions in general.

The contrast ratios (Table 3) of both gray matter and white matter with respect to lesions were significantly higher on 3D DIR images than on 3D FLAIR images $(P<.05$ and $P<.01$ for gray matter and white matter, respectively) or T2-weighted SE images $(P<.01)$. No dif- ferences were found between the sequences for contrast-to-noise ratios of lesions versus those of gray matter or white matter (Table 3). The contrast-to-noise ratio of mixed white matter-gray matter was similar for 3D DIR and T2-weighted SE images but significantly higher than that for 3D FLAIR images $(P<.05)$. The contrast-to-noise ratio of white matterCSF is, of course, very low on 3D DIR images, since the sequence was designed to suppress both white matter and CSF. Therefore, both 3D FLAIR and T2weighted SE images have significantly higher white matter-CSF contrast-tonoise ratios $(P<.01)$. The gray matter-
CSF contrast-to-noise ratio is highest at 3D FLAIR imaging but is also significantly higher at 3D DIR imaging than at T2-weighted SE imaging $(P<.01)$. The same is true for the lesion-CSF contrastto-noise ratio $(P<.01)$. All differences between contrast ratios and contrast-tonoise ratios were evaluated by using a Bonferroni-corrected Student $t$ test.

\section{DISCUSSION}

Multiple sclerosis has classically been regarded as a disorder that predominantly affects the cerebral white matter. Over the past years however, an increasing body of evidence has pointed toward the involvement of gray matter in the pathophysiology of multiple sclerosis $(4,13,15$, 25-27). This acknowledgment of gray matter involvement in the disease has led to the incorporation of (juxta)cortical lesions in recently defined multiple sclerosis diagnostic criteria $(28,29)$ and interest in the role of diffuse damage in the (normal-appearing) gray matter in determining disability and cognition $(27,30)$.

Results of our study have shown that an increased rate of detection of intracortical lesions can be obtained with 3D DIR MR imaging; that is, 3D DIR imaging has higher sensitivity than do 3D FLAIR and standard T2-weighted SE methods. Use of 3D DIR imaging showed an average increase of $152 \%$ in intracortical lesions detected per patient when compared with detection at 3D FLAIR imaging. In comparison with T2-weighted SE imaging, use of 3D DIR imaging showed a more than $500 \%$ increase in detection, which is a very important result because T2weighted SE sequences are still the most commonly employed in many clinical (and research) facilities when evaluating numbers of lesions in multiple sclerosis. This increase in intracortical lesion detection with 3D DIR imaging clearly stands out when compared with the results within the other lesion categories. Since only very few intracortical lesions were found in the control subjects, misinterpretation of artifacts for lesions seems implausible. From histopathologic studies it is known that intracortical lesions are abundant in the multiple sclerosis brain $(4,10,25)$ and that the numbers of intracortical lesions may even amount to $59 \%$ of the total lesion count (4). However, in this study, 3D DIR images depicted a mean of $4.6 \%$ of the total number of lesions to be intracortical (eight of 174 lesions). It is therefore probable that many of the intracortical lesions still cannot be visualized with 3D DIR imaging. 
Authors of several studies have pointed out the improvement in cortical and/or subcortical lesion detection with 3D FLAIR sequences in comparison with conventional SE sequences $(18,19,31)$. When the two-dimensional DIR technique was introduced $(21,22,32)$, it was reported to show advantages over twodimensional FLAIR MR when it concerned infratentorial lesions (tumorous, inflammatory and/or demyelinating, vascular) or lesions with only slightly prolonged T2 relaxation times (eg, intracortical lesions). The findings in this study of increased numbers of lesions detected at 3D DIR imaging in the cortical gray matter (lesions with low contrast on T2weighted images) are in accordance with the results of Turetschek et al (22). Although the values for the contrast-tonoise-ratio between lesion and gray matter at 3D DIR MR are similar to those at 3D FLAIR and T2-weighted SE MR imaging, the contrast between lesion and gray matter is highest at 3D DIR MR, which explains the higher conspicuity of intracortical lesions.

Besides the increased sensitivity to intracortical lesions, the second major advantage of 3D DIR imaging is its apparent potential to enable better distinction between mixed white matter-gray matter lesions, juxtacortical lesions, and purely intracortical lesions. Although T2-weighted SE MR is a sensitive technique, it may be difficult to distinguish between these three types of lesions on T2-weighted SE images. Lesions scored as juxtacortical on T2-weighted SE images often turned out to be mixed white matter-gray matter lesions on 3D DIR images. This is supported by the observation of a reduced number of juxtacortical lesions scored on 3D DIR images compared with T2-weighted SE images and an increased detection of mixed white mattergray matter lesions at 3D DIR imaging at the expense of T2-weighted SE imaging.

Previous MR studies that have been performed in an attempt to improve quantification of (juxta)cortical lesions have used two-dimensional $(18,19,31)$ and 3D FLAIR (20) MR imaging and have compared these techniques with standard T2-weighted SE imaging. In general, it was reported that 3D FLAIR imaging had an advantage over the conventional SE imaging in depicting lesions close to or in the cortex. The current study did not focus on the latter comparison, but the advantage of 3D FLAIR MR over T2weighted SE MR imaging was not as large as that in previous publications. This may be due to the increased turbo factor of the 3D FLAIR sequence used in this study, leading to shorter acquisition times at the expense of lower signal-tonoise and contrast-to-noise ratios. But even with higher signal-to-noise and contrast-to-noise ratios, 3D FLAIR has a clear disadvantage: Contrast between white matter and gray matter is poor, and, therefore, mixed white matter-gray matter lesions are difficult to distinguish from purely intracortical or juxtacortical lesions. Results of this study show that the good contrast provided on 3D DIR images allows a better assignment of lesions according to anatomic position.

Relatively small differences between techniques were found for the numbers of white matter lesions counted (periventricular and deep white matter). This means that 3D DIR can be useful for clinical or research purposes as a supplement to or maybe even as a replacement for standard T2-weighted SE and 3D FLAIR MR imaging. In other words, the increased rate of intracortical lesion detection with 3D DIR MR imaging does not come at the cost of decreases in the numbers of white matter lesions counted.

With similar total acquisition time per sequence, the $3 \mathrm{D}$ slab volumes were designed to create maximal resolution while retaining whole-brain coverage, which results in different section thicknesses (3.0 mm for T2-weighted SE, 1.25 $\mathrm{mm}$ for 3D FLAIR, and $1.8 \mathrm{~mm}$ for 3D DIR imaging). This may cause problems when attempting to match lesions observed with one sequence to those observed with another sequence. It could also be argued that the calculation of numbers of lesions may be influenced by differences in section thickness. However, the larger section thickness of T2weighted SE MR imaging is partly counteracted by the fact that the in-plane resolution of 3D DIR MR imaging is lower, which leads to similar pixel sizes, and great care was taken to avoid counting lesions twice, which is more likely to happen when the section thickness is decreased. Obviously, the section thickness of the 3D sequences could be increased to $3 \mathrm{~mm}$, which is approximately the minimum for a two-dimensional technique such as T2-weighted SE imaging. But such an approach would not properly exploit the 3D properties of 3D DIR and 3D FLAIR sequences, which have an intrinsically higher signal-to-noise ratio and a special design for acquiring thin sections.

A disadvantage of the multislab 3D DIR sequence used in this study, as well as of the multislab 3D FLAIR sequence, is its interleaved nature. This requires relatively long acquisition times (due partly to the necessary $60 \%$ oversampling in the $3 \mathrm{D}$ slab direction), is prone to subject motion between interleaved series, and introduces flow artifacts and signal intensity differences between slabs. An advantage of both 3D DIR and 3D FLAIR imaging is the (nearly) isotropic spatial resolution, which allows registration and subtraction of longitudinal images to follow disease progression (33). A future methodologic improvement is directed toward the implementation of a singleslab 3D method similar to that described for a single-slab 3D T2-weighted and FLAIR acquisition $(34,35)$. Because of the easy identification of the artifacts on 3D DIR images, diagnostic confounds will not be likely. Normal findings on 3D DIR images were previously described by Turetschek et al (22), who defined in more detail the origin of the artifacts.

Correct determination of cortical-subcortical lesion load is imperative for valid clinical and neuropsychologic studies in patients with multiple sclerosis. Results of previous studies in which FLAIR MR was used to determine (juxta)cortical lesion count or load $(14,16,36)$ have shown poor correlations with clinical disability. Accurate cortical lesion detection by use of 3D DIR imaging should be a very helpful tool in understanding more about the physical and cognitive problems encountered by patients with multiple sclerosis. The present study did not specifically focus on correlating lesion counts with clinical and neuropsychologic measures but merely showed the possible improvement in detection of intracortical lesions. It is therefore essential that future studies focus on correlating well-defined cortical-subcortical lesion load to specific measures of disability and neuropsychologic function in a larger group of patients. Moreover, follow-up studies performed to evaluate changes in cognitive functioning in relation to increasing cortical disease burden will provide useful and important information. In conclusion, 3D DIR MR imaging shows increased depiction of intracortical lesions in brains with multiple sclerosis, as well as increased definition when assessing mixed white matter-gray matter lesions.

Acknowledgments: The authors thank J. Berkhof, PhD, for statistical advice and $\mathrm{M}$. Wokke for help with the acquisition of the data.

\section{References}

1. Ferguson B, Matyszak MK, Esiri MM, Perry VH. Axonal damage in acute multiple sclerosis lesions. Brain 1997; 120:393-399. 
2. Trapp BD, Peterson J, Ransohoff RM, Rudick R, Mork S, Bo L. Axonal transection in the lesions of multiple sclerosis. N Engl J Med 1998; 338:278-285.

3. Catalaa I, Fulton JC, Zhang X, et al. MR imaging quantitation of gray matter involvement in multiple sclerosis and its correlation with disability measures and neurocognitive testing. AJNR Am J Neuroradiol 1999; 20:1613-1618.

4. Kidd D, Barkhof F, McConnell R, Algra PR, Allen IV, Revesz T. Cortical lesions in multiple sclerosis. Brain 1999; 122:17-26.

5. Rovaris M, Filippi M, Minicucci L, et al. Cortical/subcortical disease burden and cognitive impairment in patients with multiple sclerosis. AJNR Am J Neuroradiol 2000; 21:402-408.

6. Rovaris M, Filippi M. MRI correlates of cognitive dysfunction in multiple sclerosis patients. J Neurovirol 2000; 6(suppl 2):S172S175.

7. Filippi M. Linking structural, metabolic and functional changes in multiple sclerosis. Eur J Neurol 2001; 8:291-297.

8. Ge Y, Grossman RI, Udupa JK, Babb JS, Kolson DL, McGowan JC. Magnetization transfer ratio histogram analysis of gray matter in relapsing-remitting multiple sclerosis. AJNR Am J Neuroradiol 2001; 22 : 470-475.

9. Bozzali M, Cercignani M, Sormani MP, Comi G, Filippi M. Quantification of brain gray matter damage in different MS phenotypes by use of diffusion tensor MR imaging. AJNR Am J Neuroradiol 2002; 23: 985-988.

10. Brownell B, Hughes JT. The distribution of plaques in the cerebrum in multiple sclerosis. J Neurol Neurosurg Psychiatry 1962; $25: 315-320$.

11. Lumsden CE. The neuropathology of multiple sclerosis. In: Vinken PJ, Bruin GW, eds. Handbook of clinical neurology. Amsterdam, the Netherlands: Elsevier Science, 1970; 217-309.

12. Peterson JW, Bo L, Mork S, Chang A, Trapp BD. Transected neurites, apoptotic neurons, and reduced inflammation in cortical multiple sclerosis lesions. Ann Neurol 2001; 50:389-400.

13. Bo L, Vedeler CA, Nyland HI, Trapp BD, Mork SJ. Subpial demyelination in the cerebral cortex of multiple sclerosis patients. J Neuropathol Exp Neurol 2003; 62:723732.

14. Lazeron RH, Langdon DW, Filippi M, et al. Neuropsychological impairment in multiple sclerosis patients: the role of (juxta)cortical lesion on FLAIR. Mult Scler 2000; 6:280-285.
15. Rovaris M, Iannucci G, Falautano M, et al. Cognitive dysfunction in patients with mildly disabling relapsing-remitting multiple sclerosis: an exploratory study with diffusion tensor MR imaging. J Neurol Sci 2002; 195:103-109.

16. De Stefano N, Matthews PM, Filippi M, et al. Evidence of early cortical atrophy in MS: relevance to white matter changes and disability. Neurology 2003; 60:1157-1162.

17. Tubridy N, Barker GJ, MacManus DG, Moseley IF, Miller DH. Three-dimensional fast fluid attenuated inversion recovery (3D fast FLAIR): a new MRI sequence which increases the detectable cerebral lesion load in multiple sclerosis. Br J Radiol 1998; 71:840-845.

18. Moriarty DM, Blackshaw AJ, Talbot PR, et al. Memory dysfunction in multiple sclerosis corresponds to juxtacortical lesion load on fast fluid-attenuated inversion-recovery MR images. AJNR Am J Neuroradiol 1999; 20:1956-1962.

19. Bakshi R, Ariyaratana S, Benedict RH, Jacobs L. Fluid-attenuated inversion recovery magnetic resonance imaging detects cortical and juxtacortical multiple sclerosis lesions. Arch Neurol 2001; 58:742-748.

20. Tan IL, Pouwels PJ, van Schijndel RA, Ader HJ, Manoliu RA, Barkhof F. Isotropic 3D fast FLAIR imaging of the brain in multiple sclerosis patients: initial experience. Eur Radiol 2002; 12:559-567.

21. Bedell BJ, Narayana PA. Implementation and evaluation of a new pulse sequence for rapid acquisition of double inversion recovery images for simultaneous suppression of white matter and CSF. J Magn Reson Imaging 1998; 8:544-547.

22. Turetschek K, Wunderbaldinger $P$, Bankier AA, et al. Double inversion recovery imaging of the brain: initial experience and comparison with fluid attenuated inversion recovery imaging. Magn Reson Imaging $1998 ; 16: 127-135$.

23. Pouwels PJ, Kuijer JP, Korf ES, Geurts JJ, Barkhof F. 3D-double inversion recovery for high resolution gray matter brain imaging (abstr). In: Proceedings of the 10th Meeting of the International Society for Magnetic Resonance in Medicine. Berkeley, Calif: International Society for Magnetic Resonance in Medicine, 2002; 1290.

24. Poser CM, Paty DW, Scheinberg L, et al. New diagnostic criteria for multiple sclerosis: guidelines for research protocols. Ann Neurol 1983; 13:227-231.

25. Bo L, Vedeler CA, Nyland H, Trapp BD, Mork SJ. Intracortical multiple sclerosis lesions are not associated with increased lymphocyte infiltration. Mult Scler 2003; 9:323-331.
26. Ge Y, Grossman RI, Udupa JK, Babb JS, Mannon LJ, McGowan JC. Magnetization transfer ratio histogram analysis of normal-appearing gray matter and normal-appearing white matter in multiple sclerosis. J Comput Assist Tomogr 2002; 26:62-68.

27. Miller DH, Thompson AJ, Filippi M. Magnetic resonance studies of abnormalities in the normal appearing white matter and grey matter in multiple sclerosis. J Neurol 2003; 250:1407-1419.

28. Barkhof F, Filippi M, Miller DH, et al Comparison of MRI criteria at first presentation to predict conversion to clinically definite multiple sclerosis. Brain 1997; 120:2059-2069.

29. McDonald WI, Compston A, Edan G, et al. Recommended diagnostic criteria for multiple sclerosis: guidelines from the International Panel on the Diagnosis of Multiple Sclerosis. Ann Neurol 2001; 50:121-127.

30. Cercignani $M$, Bozzali $M$, Iannucci $G$, Comi G, Filippi M. Magnetisation transfer ratio and mean diffusivity of normal appearing white and grey matter from patients with multiple sclerosis. J Neurol Neurosurg Psychiatry 2001; 70:311-317.

31. Boggild MD, Williams R, Haq N, Hawkins $\mathrm{CP}$. Cortical plaques visualised by fluidattenuated inversion recovery imaging in relapsing multiple sclerosis. Neuroradiology 1996; 38(suppl 1):S10-S13.

32. Redpath TW, Smith FW. Technical note: use of a double inversion recovery pulse sequence to image selectively grey or white brain matter. Br J Radiol 1994; 67: $1258-1263$.

33. Tan IL, van Schijndel RA, Pouwels PJ, Ader HJ, Barkhof F. Serial isotropic three-dimensional fast FLAIR imaging: using image registration and subtraction to reveal active multiple sclerosis lesions. AJR Am J Roentgenol 2002; 179:777-782.

34. Mugler JP 3rd, Bao S, Mulkern RV, et al. Optimized single-slab three-dimensional spin-echo MR imaging of the brain. Radiology 2000; 216:891-899.

35. Zhao L, Mugler JP, Wei L, Chen N, et al. A high-resolution clinical whole-brain scan using single-slab three-dimensional T1W, T2W, and FLAIR fast spin-echo sequences (abstr). In: Proceedings of the 10th Meeting of the International Society for Magnetic Resonance in Medicine. Berkeley, Calif: International Society for Magnetic Resonance in Medicine, 2002; 1294.

36. Ciccarelli O, Brex PA, Thompson AJ, Miller DH. Disability and lesion load in MS: a reassessment with MS functional composite score and 3D fast FLAIR. J Neurol 2002; 249:18-24. 